\title{
Possible space flight-induced catecholamine cardiomyopathy: Neil Armstrong's last 20 lunar minutes
}

This article was published in the following Dove Press journal:

Research Reports in Clinical Cardiology

17 January 2014

Number of times this article has been viewed

\section{William J Rowe}

Former Assistant Clinical Professor of Medicine, Medical University of Ohio at Toledo, Ohio, USA
Correspondence: William J Rowe I 485 Bremerton Lane, Keswick, VA 22947, USA

Email rowerun@aol.com

\begin{abstract}
The hypothesis underpinning this paper is that space flight may predispose to catecholamine cardiomyopathy. Catecholamine levels in space are twice those of the supine levels on Earth. Serum magnesium levels are significantly reduced, with potential vicious cycles triggered by elevation of catecholamines. These are conducive to coronary vasospasm with clot formation from oxidative stress and calcium overload, and ultimately, temporary impairment of left ventricular function could occur. Experimental animals in space have shown a significant increase in norepinephrine levels with various microcirculatory disorders and serious myocardial pathology. During extravehicular activity (space walks), astronauts show heart rates of 150-174 beats per minute. Before exposure to the iron-laden dust brought into the habitat on his space suit, Neil Armstrong's heart rates on the lunar surface were 130-160 beats per minute, and accompanied by dyspnea on two occasions during his last 20 minutes on the moon. A stress test done on the day after splashdown was consistent with "ischemic left ventricular dysfunction". To support this hypothesis, echocardiography on the international space station might show left ventricular hypokinesia. Alpha adrenergic blockade, correction of invariable significant magnesium deficits, along with correction of invariable atrial natriuretic peptide deficits, may counteract the vasoconstrictive action of norepinephrine.
\end{abstract}

Keywords: space flight, catecholamines, cardiomyopathy, magnesium, oxidative stress, heat intolerance, calcium

\section{Introduction}

It is conceivable that space flight with microgravity and hypokinesia (diminished movement) can lead to catecholamine cardiomyopathy. This hypothesis was generated by recent data obtained from the National Aeronautics and Space Administration through the Freedom of Information Act, showing two critical findings, ie, dyspnea and tachycardia in Neil Armstrong, the world's first moon walker. Although I have wondered about this association for several years, I did not have the courage to publish this association until these vitally important data were obtained. We must keep in mind that with only about 530 humans ever having been in space and with only 12 moon walkers, every shred of data must be carefully evaluated as to its importance, given it may be a century or more before worthwhile epidemiologic studies can be published.

Relatively little has been accomplished in providing solutions. I have shown that high levels of catecholamines and decreased levels of magnesium ions, with vicious cycles generated between the two, can trigger permanent myocardial injury. Similarly, with severe ischemia, there can be a vicious cycle with high catecholamines, with mechanisms related to coronary vasospasm. ${ }^{1-6}$ 
Catecholamine elevations and their products have been postulated to trigger an influx of calcium intracellularly. Magnesium is both a powerful antioxidant and a calcium blocker. Insulin is also an antioxidant, and since both magnesium and insulin are required for the synthesis of transferrin, ${ }^{7}$ keeping iron out of harm's way from hydroxyl radicals. In the presence of a magnesium deficit, insulin resistance can develop, along with endothelial dysfunction (Figure 1). An example of space-related systemic endothelial dysfunction is described in my previous paper titled "Apollo 15 space syndrome", ${ }^{4}$ that was vindicated several years ago when data were obtained from the National Aeronautics and Space Administration through the Freedom of Information Act showing that, on the day of return, James Irwin, lunar module pilot, of the 12 day Apollo 15 mission, had "cyanosis of the nail beds". I have never seen this during a stress test, despite having supervised over 5,000 hospitalbased, symptom-limited maximal Bruce treadmill stress tests. However, this event occurred on return from the moon, and was therefore confounded by Irwin's exposure to iron-laden lunar dust. $^{8}$ On the other hand, there was no confounding by lunar dust associated with the severe fingertip pain experienced by Irwin and David Scott, commander of the Apollo 15 mission, while on the lunar surface before entry into the lunar module, bringing highly toxic, iron-laden dust into the habitat on their space suits and inhaling this dust all the way back to Earth. ${ }^{4}$ Further, in his autobiography, Irwin stresses that this pain was unexpected, given that they did not experience any such severe discomfort when training in space suits underwater. ${ }^{9}$
Stress cardiomyopathy may be a form of myocardial stunning induced by a surge of catecholamines with acute reversible apical left ventricular dysfunction. ${ }^{10-14}$ Calcium overload in myocardial cells may produce left ventricular dysfunction in this situation.

In order to better understand the complexities of catecholamine elevations and their relationship with magnesium in space, my experience with studies of two extraordinary world class ultramarathoners should be helpful. This began with my study of Sy Mah in 1987 for a paper. I had been invited to present in the People's Republic of China, as a member of the North American Fitness Delegation. Mah was listed in the Guinness Book of Records for having completed 524 marathons. Ten months after I studied him with a symptom-limited maximum treadmill stress test, on which he performed surprisingly poorly, Mah died of complications from lymphoma, at age 62 years. I sent his heart to William Roberts, arguably the world's leading cardiac pathologist, at the National Institutes of Health, asking whether there were any findings suggestive of a pheochromocytoma. His report indicated that there was nothing specific which would meet these criteria, but there was focal fibrosis in the papillary muscles. This led to my hypothesis that extraordinary unremitting endurance exercise can induce permanent injury in a perfectly normal heart. When Mah was studied at autopsy, his coronaries appeared normal in terms of atherosclerosis. At the same time, I also studied Mary Hanudel, ${ }^{15}$ the 1987 female US ultramarathoner of the year. Her treadmill stress

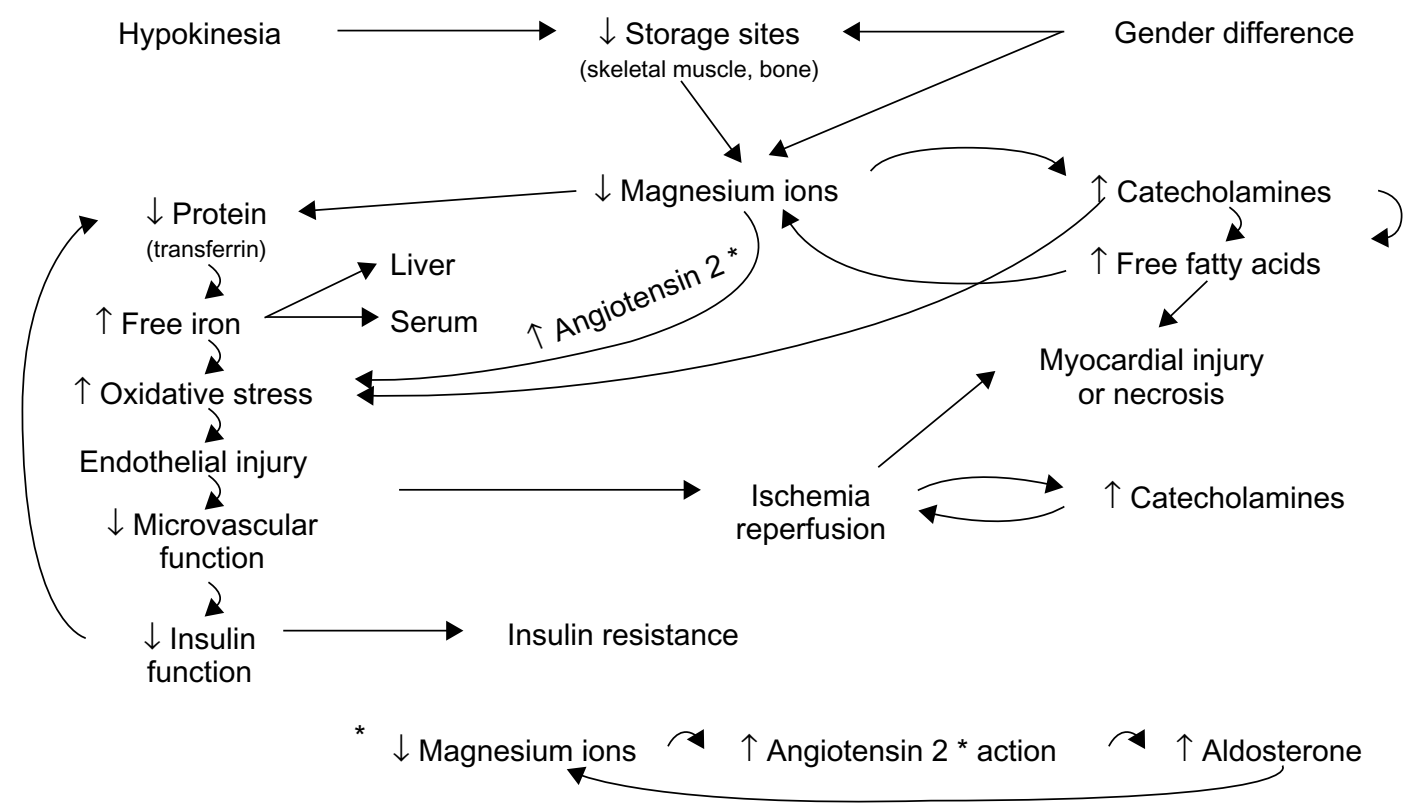

Figure I Four vicious cycles complicating hypokinesia of space flight.

Notes: Rowe WJ. The case for a subcutaneous magnesium product and delivery device for space missions. J Am Coll Nutr. 2004;23:525-528S. ${ }^{2}$ C2004, William J, Rowe MD. All rights reserved. 
test performance was also surprisingly poor, and she was unable to complete stage 5 of the Bruce protocol. ${ }^{16}$ The explanation was provided the next day, when it was found that her blood count was half the normal level, stress-related, complicating unrecognized chronic gastrointestinal bleeding, possibly from a stress ulcer. Of importance was the fact that both runners showed evidence of a magnesium deficiency, using a magnesium loading test ${ }^{17}$ since a normal serum test is insensitive; $99 \%$ of magnesium is intracellular.

I have stressed the fact that even a brief lunar mission of 12 days (as was the case for Irwin on Apollo 15), can trigger numerous cardiovascular complications without the confounder of radiation-induced oxidative stress. On the Apollo missions, there was no significant radiation. ${ }^{18}$ In vitro, animal, and human studies indicate that the endothelium is vulnerable to space flight-related dysfunction injury, with magnesium playing a central role, and there is loss of magnesium storage sites in skeletal muscle and bone as a complication of hypokinesia. Further, I believe that it is impossible to offset this with exercise in the presence of microgravity because both too much and too little exercise can injure a perfectly normal heart; in both conditions, telomeres, which are necessary to maintain the integrity of chromosomes, are shortened. ${ }^{19}$ Cyclic GMP is a second messenger for two vitally important vessel dilators and clot busters, ie, atrial natriuretic peptide and nitric oxide, that are necessary for endothelial function; after 5 months in low orbit on the Mir space station, cyclic GMP is no longer detectable. ${ }^{20}$

In space, there is a loss of skeletal muscles within a couple of weeks, and skeletal muscle is a storage site for magnesium. In 6 months, $40 \%$ of the soleus muscle is lost, with the aging process accelerated by a factor of $10 .{ }^{1}$ In addition, $1 \%-2 \%$ of the magnesium stored in bone is lost each month, and further magnesium reduction occurs with invariable malabsorption. With the reduction in magnesium ions, there are elevations in catecholamines, creating a vicious cycle conducive to catecholamine cardiomyopathy. Such a vicious cycle can induce oxidative stress, elevated inflammatory cytokines, and insulin resistance that can, in turn, precipitate self-sustaining vascular inflammation (Figure 1).

Space flight-related thrombocytopenia is probably responsible for the reduction in vascular endothelial growth factor (VEGF), given that platelets are the primary source of VEGF. Both magnesium and VEGF regulate endothelial function and repair and are required for angiogenesis. In the presence of space flight-related insulin resistance, there would also be diminished VEGF expression. ${ }^{2}$ In addition to the aforementioned vicious cycles related to space flight, another is triggered by the elevation of inflammatory cyto kines complicating a magnesium ion deficit, which in turn leads to further loss of skeletal muscle reservoirs. Elevated cytokine (tumor necrosis factor alpha) levels have also been shown to complicate sleep deprivation, the average duration of sleep during space flight is 6 hours. This is also conducive to oxidative stress. Further, the calcium-blocking effect of magnesium may serve an even a more important function, since it has been postulated that, with elevations in carbon dioxide as demonstrated on the Mir space station, there may also be an intracellular shift of calcium. ${ }^{20}$

One of the reasons why magnesium is such a powerful antioxidant is that it is necessary for the synthesis of transferrin, as is insulin. ${ }^{2}$ Magnesium binds transferrin tightly to iron, which is conducive to elevated levels of hydroxyl ions. ${ }^{21}$ The antioxidant effect of magnesium could potentially offset the auto-oxidation of catecholamines and the effects of $100 \%$ oxygen for 3 hours prior to the early Apollo missions. Further, $100 \%$ oxygen is required for more than 2 hours prior to a space walk to wash out nitrogen bubbles, thereby preventing decompression sickness, with space walks lasting up to 8 hours and therefore potentially very dangerous.

When I presented my first space-related paper at a National Aeronautics and Space Administration Space Conference (Eleventh IAA Man In Space Symposium, Toulouse, France) in 1995, there were very strong objections to my insistence that catecholamines must be elevated in space. I supported my arguments with animal studies from Russia ${ }^{22}$ and my own publications regarding the vicious cycles I have described. Although the moderator estimated that it would require 20 years of research to determine the importance of catecholamine elevations, in just a few years it was concluded that "baseline sympathetic outflow, however measured, is higher in space than on Earth". ${ }^{23,24}$ In fact, it has been shown that the catecholamine levels are twice those in the supine position on Earth. ${ }^{24} \mathrm{I}$ believe that the mechanism involved is primarily related to this invariable deficit of magnesium. Even though the sensitivity of serum magnesium is extremely poor, with $99 \%$ of magnesium being intracellular, in large groups of astronauts and cosmonauts, serum magnesium levels are reduced $(P<0.0001){ }^{25}$

\section{Animal studies}

A significant increase in the concentration of norepinephrine in heart tissue was found in experimental rats after a flight on the Kosmos 936 biosatellite. ${ }^{22}$ A nonuniform distribution of epinephrine and norepinephrine in the ventricles was also observed in these animals after a flight on the Kosmos 
1129 biosatellite. In many tissues, including cardiac muscle, there were changes in the structure of the mitochondria, and a reduced rate of ATP synthesis due to a lesser degree of conjugation of oxidative phosphorylation. In cardiac muscle, there were pronounced atrophic and dystrophic changes, a decrease in the size and integrity of muscle fiber structure, and suppressed synthesis of myocardial proteins, as well as evidence of impairment in the repair mechanism. ${ }^{22}$

\section{Astronaut studies}

In addition to the Russian animal studies cited above, the following is provocative: "The crewmen of the second expedition aboard Salut 4 demonstrated elevated activity of the medullary layer of the adrenal glands (higher level of excretion of norepinephrine)." Further, "A space flight offers a whole multitude of situations stimulating a sharp release of catecholamines". ${ }^{22}$ Heart rates of $150-174$ beats per minute are frequently recorded during space walks. ${ }^{25}$ The information I received from the National Aeronautics and Space Administration through the Freedom of Information Act showed that Neil Armstrong, at the age of 39 years, apparently complained of dyspnea on two occasions during the last 20 minutes of his brief lunar excursion, and that this symptom occurred prior to exposure to the dust carried into the lunar module on his space suit (Figure 2A). This symptom is very important given that some individuals experience dyspnea and pulmonary edema with stress cardiomyopathy. Plasma catecholamine concentrations have been shown to be 2-3 times higher in patients with stress cardiomyopathy than in patients hospitalized with an acute myocardial infarction. ${ }^{10}$

Armstrong's heart rate during these last 20 lunar minutes was high, ranging between 130 and 160 per minute (Figure 2A). On the lunar surface, at one-sixth gravitational forces, his tachycardia and dyspnea were what one would expect with running, whereas just before splashdown, at the time of "separation", his heart rate fell surprisingly to only 61 beats per minute (Figure $2 \mathrm{~B})$. On the day of landing $(\mathrm{R}+0)$, his electrocardiogram was normal, showing a heart rate of 58 beats per minute. However, his stress test on the day after splashdown was consistent with "ischemic left ventricular dysfunction", with a specificity of $100 \% .^{3}$

\section{Hypothesis-supporting studies}

With the release of Armstrong's data, there is now sufficient evidence to postulate a space flight-induced catecholamine cardiomyopathy triggering dyspnea, that occurred in Armstrong's case without the confounding factor of iron-laden dust inhalation. Catecholamine cardiomyopathy is a temporary condition, often described as "self-limiting". Studies of this would be required on the international space station, and could be done with echocardiography, looking for global or localized hypokinesia of the left ventricle, ie, an inverted Takotsubo pattern. There would be apical suppression with basal sparing in stress cardiomyopathy. Treatment with an alpha-adrenergic blocking agent for several weeks may offset these abnormalities. Also, subcutaneous magnesium would be required using a replenishable silicon subcutaneous device (as yet not developed) to correct invariable significant magnesium reductions. Further, it would be necessary to correct the invariable $10 \%$ reduction of plasma volume thought to be precipitated by leaks in the endothelium and compounded by a reduction in the thirst mechanism. Correction of reduced atrial natriuretic peptide levels, which can decrease by over $40 \%$ in 7-12 days, would be required. Atrial natriuretic peptide may act to counteract the vasoconstrictive action of norepinephrine. However, given that magnesium is necessary for the synthesis and release of atrial natriuretic peptide, ${ }^{1,26}$ correcting the invariable magnesium depletion may suffice.

I had been puzzled by the fact that whereas Armstrong's heart rate during these last 20 lunar minutes ranged from 130-160 beats per minute, at the time of separation with deployment of the parachute for splashdown, his heart rate fell to a normal resting level of 61 beats per minute (Figure 2B). Since it takes 3 days to return to Earth, the most likely explanation was that he restored his plasma volume to a considerable level. The combination of decreased thirst and considerable sweating triggered by decreased convection would be responsible. On the moon at noontime the temperature reaches $250^{\circ} \mathrm{F}$. It has been stressed by Merli et $\mathrm{al}^{27}$ that since their studies show mid-cavity dynamic obstruction of the left ventricle, with rehydration and a fall in catecholamine levels the interventricular gradient resolves and distal function recovers.

\section{Discussion}

In addition to the oxidative stress arising from use of $100 \%$ oxygen prior to lift-off during the early Apollo missions, as noted previously, astronauts trained in "intense summer heat". This condition, along with tachycardia, is a deadly combination. Tachycardia can enhance oxidative stress. ${ }^{28}$ Superoxide anions and hyperoxia inactivate nitric oxide, ${ }^{29}$ and this combination may trigger sudden death. ${ }^{30}$ Further, adrenergic-related sinus tachycardia may precede a lethal arrhythmia.

It is of considerable interest that up to $90 \%$ of cases of catecholamine cardiomyopathy have been reported in women 
A

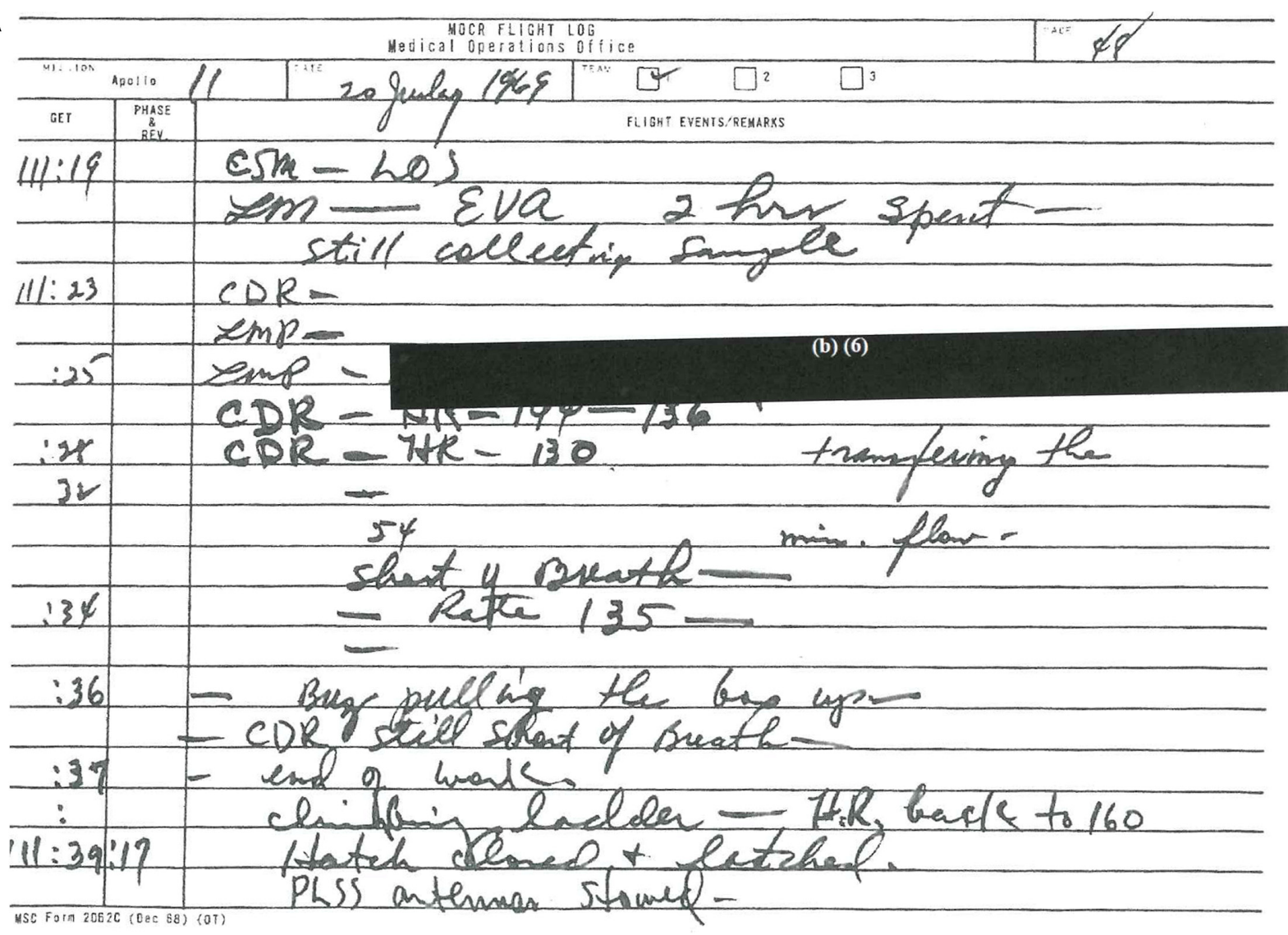

B

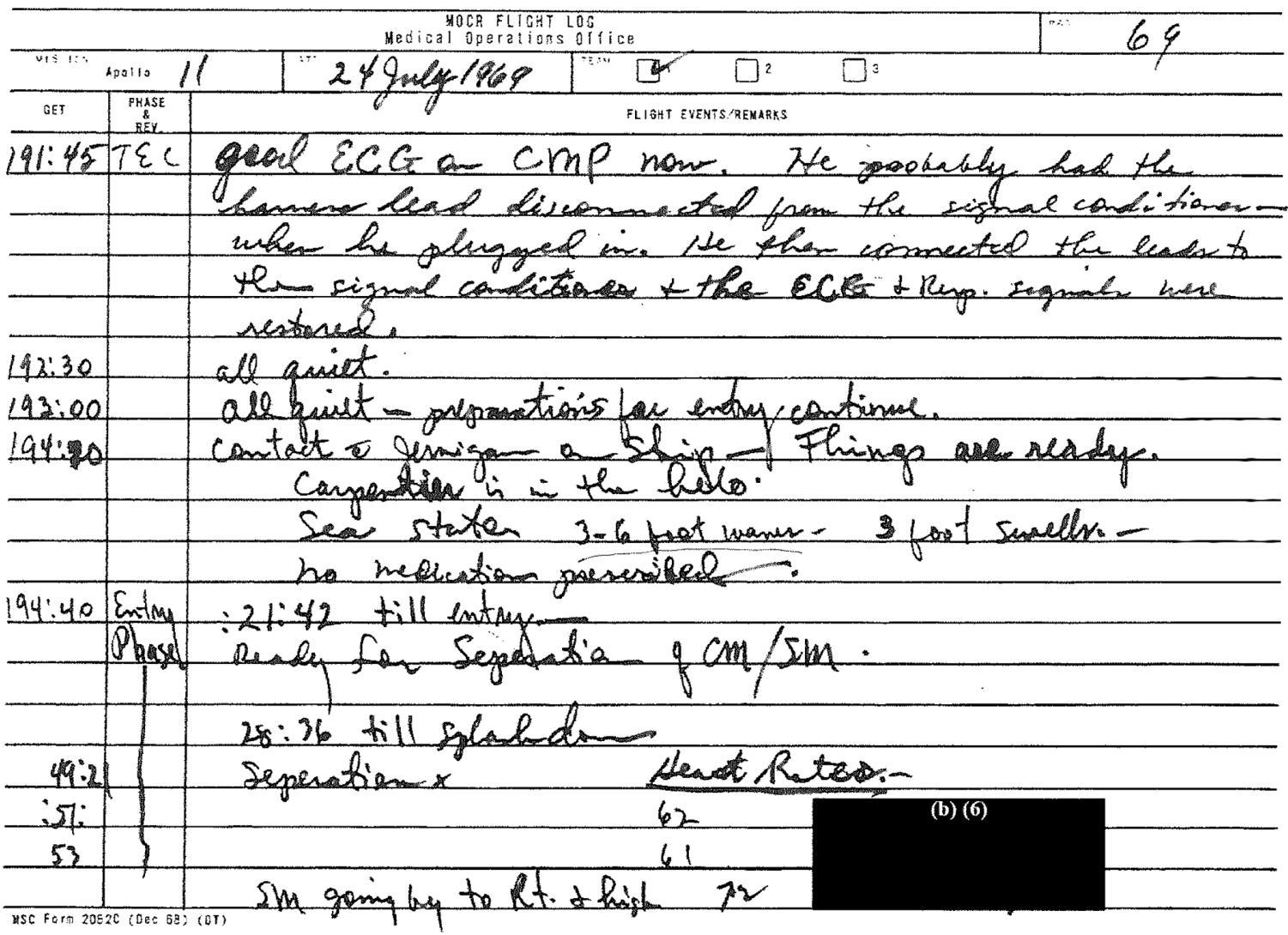

Figure 2 Historical document showing remission of severe tachycardia after a 3 day return to Earth. 
(most commonly in their postmenopausal years), but with no clear explanation. It is conceivable that the mechanisms on Earth may be similar to those which apply to increased core body temperature with exercise in space, first described in 1995 (personal communication, A Hansson). With decreased blood flow to the periphery, in order to lose heat by convection, in both conditions there is a predisposition for excessive sweating, leading in turn to excessive loss of magnesium. There is also magnesium loss through the kidneys with increased angiotensin and in turn increased aldosterone. ${ }^{1}$ Magnesium appears to play a significant role in heat acclimation. ${ }^{31}$ In the presence of a magnesium deficiency, there is an increased angiotensin effect. Angiotensin II induces catecholamine release by direct ganglionic excitation. ${ }^{32}$ This is conducive to increased free fatty acid levels, decreased numbers of magnesium ions, and another vicious cycle. If the calcium to magnesium intake ratio is too high, ie, above the ideal range of $2: 1$, this would lead in turn to impaired magnesium absorption, an increased potential for clotting with vasospasm, and further oxidative stress. ${ }^{6}$ This may be more likely in women taking excessive calcium supplements and in space possibly incorrect attempts to ward off osteoporosis.

In space, there is a $1 \%-2 \%$ loss of bone each month, and $60 \%$ of magnesium is stored in bone. The ideal calcium to magnesium intake ratio has not been established, but could be determined by rat studies on the international space station, using methods similar to those described previously. ${ }^{1}$ The higher incidence of catecholamine cardiomyopathy in postmenopausal women may be because ovariectomy in animal models leads to an increased sensitivity to vasoconstrictive agents with reduced relaxation of blood vessels and therefore less peripheral blood flow. Loss of estrogen increases levels of angiotensin, which induces catecholamine release and further vasoconstriction. ${ }^{33}$ Studies of postmenopausal women might assist us to understand these space-related complications further, particularly during exercise. ${ }^{1}$

The mechanisms of heat intolerance with exercise in space are similar to those in postmenopausal women, with both groups being vulnerable to catecholamine cardiomyopathy. However, an example in Irwin's autobiography points out the particular vulnerability of moon walkers. Irwin (Apollo 15) emphasizes that when he and Scott returned from their first lunar excursion, before potential confounding by inhalation of toxic dust, "perspiration poured from their gloves". ${ }^{8,9}$ His heart rate while configuring the lunar rover reached 167 beats per minute, while at the same time Scott's was 130 beats per minute. ${ }^{18}$ During the 4 minute re-entry, at $7 \mathrm{G}$ forces, Irwin experienced symptoms consistent with classical angina along with symptoms of congestive heart failure, with such intense dyspnea that he was unable to deliver the verbal recording needed during the radio blackout; ${ }^{9}$ however, here there was the confounding factor of dust inhalation, which was not present during Armstrong's repeated experience of dyspnea.

Finally, because of the National Aeronautics and Space Administration's restrictive policy that an astronaut must be deceased before information can be released to the public through the Freedom Of Information Act, it is indeed a tragedy that at least two decades of potential research regarding this condition, first described in 1990, have been lost. If this very preliminary description is supported by research on the international space station, it may be that 5 days in microgravity is sufficient to trigger temporary impairment in cardiac function. More data is needed to determine if there is such an effect during a very prolonged space mission. Will we, despite its extreme complexity, require gene therapy or can we duplicate earth's G-force when exploring over long distances or building habitats on the moon, asteroids, or Mars?

\section{Disclosure}

This work was presented in part at the Fourth World Gene Convention in 2013 and also at the International Drug Discovery Science and Technology Therapy meeting, November 13-16, 2013, Haikou, People's Republic of China. The author reports no other conflicts of interest in this work.

\section{References}

1. Rowe WJ. Correcting magnesium deficiencies may prolong life. Clin Interv Aging. 2012;7:51-54.

2. Rowe WJ. The case for a subcutaneous magnesium product and delivery device for space missions. J Am Coll Nutr. 2004;23:525S-528S.

3. Rowe WJ. Moon walkers and urban pollution. Am J Med. 2013;126: e1-e2.

4. Rowe WJ. The Apollo 15 space syndrome. Circulation. 1998;97: 119-120.

5. Rowe WJ. Extraordinary unremitting endurance exercise and permanent injury to normal heart. Lancet. 1992;340:712-714.

6. Rowe WJ. Calcium-magnesium-ratio intake and cardiovascular risk. Am J Cardiol. 2006;98:140.

7. Rowe WJ. Space flight-related endothelial dysfunction with potential congestive heart failure. Proceedings of the eighth world congress on heart failure, mechanisms and management. Washington DC, July 13-16 2002.

8. Jomova K, Valko M Advances in metal -induced oxidative stress and human disease. Toxicology. 2011;283:65-87.

9. Irwin JB, Emerson WA. To Rule the Night. The Discovery Voyage of Astronaut Jim Irwin. Philadelphia, PA: AJ Holman Co; 1973.

10. Kassim TA, Clarke DD, Mai VQ, Clyde PW, Shakir KMM. Catecholamine-induced cardiomyopathy. Endocr Pract. 2008;14: 1137-1149.

11. Lyon AR, Rees PS, Prasad S, Poole-Wilson PA, Harding SE. Stress (Takotsubo) cardiomyopathy - a novel pathophysiological hypothesis to explain catecholamine-induced acute myocardial stunning. Nat Clin Pract Cardiovasc Med. 2008;5:22-29. 
12. Akashi YJ, Goldstein DS, Barbaro G, Ueyama T. Takotsubo cardiomyopathy: a new form of acute, reversible heart failure. Circulation. 2008;118:2754-2762.

13. Nef HM, Mollmann H, Akashi YJ, Hamm CW. Mechanisms of stress (Takotsubo) cardiomyopathy. Nat Rev Cardiol. 2010;7:187-193.

14. Hurst RT, Prasad A, Askew JW, Sengupta PP, Tajik AJ. Takotsubo cardiomyopathy: a unique cardiomyopathy with variable ventricular morphology. JACC Cardiovasc Imaging. 2010;3:641-649.

15. Rowe WJ. Our ancestors had it right. Am J Cardiol. 2000;86:256.

16. Shah BN On the 50th anniversary of the first description of a multistage exercise treadmill test: re-visiting the birth of the Bruce Protocol. Heart. 2013;99:1793-1794.

17. Gullestad L, Midvedt K, Dolva LO, Norseth J, Kjekshus J. The magnesium loading test: reference value in healthy subjects. Scand J Clin Invest. 1994;54:23-31.

18. National Aeronautics and Space Administration. Apollo 15 mission report: MSC 05161. Houston, TX: National Aeronautics and Space Administration; 1971.

19. Ludlow AT, Zimmerman JB, Witkowski S, Hearn JW, Hatfield BD, Roth SM. Relationship between physical activity level, telomere length, and telomerase activity. Med Sci Sports Exerc. 2008;10:1764-1771.

20. Rossler A, Noskov V, Laszlo Z, Polyakow VV, Hinghoffer-Szalkay HG. Permanent depression of plasma cGMP during long-tem space flight. Physiol Res. 2001;50:83-90.

21. Parent ME, Zemel MB. Magnesium potentiation of iron-transferrin binding. Life Sci. 1989;44:1007-1012.

22. Atkov O, Bednenko VS. Hypokinesia and Weightlessness: Clinical and Physiologic Aspects. Madison, WI, USA: International Universities Press; 1992.
23. Eckberg DL; Neurolab Autonomic Nervous System Team. Bursting into space: alterations of sympathetic control by space travel. Acta Physiol Scand. 2003;177:299-311.

24. Christensen NJ, Drummer C, Norsk P. Renal sympathoadrenal responses in space. Am J Kidney Dis. 2001;38A:679-683.

25. Katunysev VP, Osipov YY, Barer AS, Gnoevaya NK, Tarasenkov GG. The main results of EVA medical support on the Mir space station. Acta Astronaut. 2004;54:577-583.

26. Brenner BM, Ballerman BJ, Gunning ME, Zeidel MI. Diverse biological actions of atrial natriuretic peptide. Physiological reviews., 1990; 70:665-699.

27. Merli E, Sutcliffe S, Gori M, Sutherland GGR. Tako-Tsubo cardiomyopathy: new insights into the possible underlying pathophysiology. Eur J Echocardiography. 2006;7:53-61.

28. Yamamoto E, Zhong-Fang L, Yamashita T, et al. Enhancement of cardiac oxidative stress by tachycardia and its critical role in cardiac hypertrophy and fibrosis. J Hypertens. 2006;24:2057-2069.

29. Rubanyi GM, Vanhoutte PM. Superoxide anions and hyperoxia inactivate endothelium-derived factor. Am J Physiol. 1986;250:H822-H827.

30. De Luna AB, Coumel P, Leciercq JF. Ambulatory sudden cardiac death; mechanisms of production of fatal arrhythmia on the basis of data from 157 cases. Am Heart J. 1989;117:151-159.

31. Stendig-Lindberg G, Moran D, Shapiro Y. How significant is magnesium in thermoregulation? J Basic Clin Physiol Pharmacol. 1998;9:73-85.

32. Dendorfer A, Thornagel A, Raasch W, Grisk O, Tempel K, Dominisk P. Angiotensin 2 induces catecholamine release by direct ganglionic excitation. Hypertension. 2002;40:348-354.

33. Stice JP, Lee JS, Pechenino S, Knowlton AA. Estrogen, aging and the cardiovascular system. Future Cardiol. 2009;5:93-203.
Research Reports in Clinical Cardiology

\section{Publish your work in this journal}

Research Reports in Clinical Cardiology is an international, peerreviewed, open access journal publishing original research, reports, editorials, reviews and commentaries on all areas of cardiology in the clinic and laboratory. The manuscript management system is completely online and includes a very quick and fair peer-review system.

\section{Dovepress}

Visit http://www.dovepress.com/testimonials.php to read real quotes from published authors. 